Conclusions Our study shows that there are differences in exposure to carcinogenic agents among shift and non-shift workers, and so there is a need for prevention programs in order to reduce these discrepancies.

\section{A.4 THE RELATIONSHIP BETWEEN SHIFT WORK AND SLEEP DISTURBANCE IN HOSPITAL WORKERS}

${ }^{1}$ Dong-Hee Koh*, ${ }^{2}$ Ju-Hyun Park, ${ }^{3}$ Seo-Hyun Yoon, ${ }^{4}$ Hyun-Joo Kim. ${ }^{1}$ Department of Occupational and Environmental Medicine, Int St. Mary's Hospital, Incheon, South Korea; ${ }^{2}$ Department of Statistics, Dongguk University, Seoul, South Korea; ${ }^{3}$ Ewha Medical Research Institute, Ewha Womans University School of Medicine, Seoul, South Korea; ${ }^{4}$ Department of Occupational and Environmental Medicine, Ewha Womans University Mokdong Hospital, South Korea

\subsection{6/OEM-2019-EPI.88}

Introduction Many hospital workers suffer from sleep disturbance due to shift work. The best way to mitigate the problem would be reducing night shift. However, that is commonly impossible in reality. Thus, we aimed to examine the factors associated with sleep disturbance and suggest better-rotating schedules for hospital workers.

Methods We used sleep questionnaires asking sleep disturbance including sleep onset and maintenance problems for measuring health outcomes. Also, We obtained a duty schedule of the workers for assessing exposure. We examined the effect of the pattern of shift schedule and amount of night shifts on sleep disturbance.

Results A total of 590 workers participated in this study. Female (95\%) 3-rotating shift (92\%) workers dominated. For workshift pattern, two consecutive night shifts increased the risk of sleep maintenance problem significantly. For the amount of night shift, two or more night shifts per week increased the risk of sleep onset problem significantly.

Conclusion Our results suggest that avoding consecutive night shifts and restricting night shift to one time per week would be helpful to prevent sleep disturbance in hospital workers.

\section{A.5 NIGHT SHIFT WORK IS ASSOCIATED WITH ABNORMAL LIVER FUNCTION}

${ }^{1}$ Lap Ah Tse*, ${ }^{1}$ Feng Wang, ${ }^{2}$ Liuzhuo Zhang, ${ }^{2}$ Zhimin Li. ${ }^{1}$ The Chinese University of Hong Kong, Shatin, Hong Kong; ${ }^{2}$ Shenzhen Prevention and Treatment Center for Occupational Diseases, Shenzhen, China

\subsection{6/OEM-2019-EPI.89}

Objective To examine the relationship between night shift work and elevated level of alanine transaminase (e-ALT) of workers, stratified by status of non-alcohol fatty liver.

Methods We established a prospective cohort of night shift workers in Shenzhen, China. All 4740 male workers who had liver function data from the baseline survey of this night shift workers' cohort were included in the current analysis. We collected information on demographics, lifestyles, and lifetime working schedule from standardized questionnaire. We examined workers' NAFL status using liver sonography and their serum ALT levels using the automatic biochemical analyzer. Unconditional logistic regression models were used to evaluate odds ratios (ORs) and 95\% confidence interval $(95 \% \mathrm{CI})$.
Results This cohort contained 39.5\% night shift workers. Compared with the day workers, night shift workers had higher odds of e-ALT (OR: 1.18; 95\% CI 1.00-1.40). Stratified analysis showed that a positive gradient between night shift years and elevated e-ALT was only restricted to the night shift workers without NAFL. No similar trend was observed among those with NAFL.

Conclusions Night shift work is positively associated with the abnormal liver function in workers without NAFL, which indicates that shift work involving circadian disruption is likely to affect abnormal liver function.

[National Natural Science Foundation of China (Project number 81273172 and 81372964), Shelly@cuhk.edu.hk (Lap Ah Tse)]

\section{A.6 OCCUPATIONAL EXPOSURE TO EXTREMELY LOW FREQUENCY MAGNETIC FIELDS AND MELATONIN IN MALE ROTATING SHIFT WORKERS} ${ }^{1}$ Michelle C Turner* ${ }^{*}{ }^{1}$ Esther Gracia-Lavedan, ${ }^{1}$ Gemma Castaño-Vinyals, ${ }^{2}$ Debra J Skene,
${ }^{2}$ Benita Middleton, ${ }^{1}$ Elisabeth Cardis, ${ }^{1}$ Manolis Kogevinas. ${ }^{1}$ Barcelona Institute for Global
Health (ISGlobal), Barcelona, Spain; ${ }^{2}$ Chronobiology, University of Surrey, Guilford, UK

\subsection{6/OEM-2019-EPI.90}

Background Occupational exposure to extremely low frequency magnetic fields (ELF-MF) is a suspected cancer risk factor, however, the epidemiological literature is limited and potential biological mechanisms unclear. Night shift work and associated circadian misalignment, leading to decreased melatonin production is also suspected in cancer. It has been suggested that ELF-MF exposure may decrease melatonin production leading to the promotion of cancer development, however previous findings are inconsistent. We assessed ELFMF exposure and melatonin production in a recent study of male rotating shift workers.

Methods A total of 50 male shift workers rotating in a 3 week night, 3 week evening, and 3 week morning schedule were recruited at a local car manufacturing facility. Personal full-shift measurements of ELF-MF exposure were performed (EMDEX II, 40-800 Hz) and urine samples from all voids over 24 hour collected on two working days (end of 3 week night and end of 3 week morning shifts). Urinary concentrations of 6-sulfatoxymelatonin (aMT6s), the main melatonin metabolite, were measured in all samples (creatinine corrected), with the mesor ( 24 hour mean) derived based on cosinor models.

Results Arithmetic mean (AM (SD)) and geometric mean (GM (GSD)) ELF-MF levels were $0.05(0.06) \mu \mathrm{T}$ and 0.03 (0.02) $\mu \mathrm{T}$, respectively. Peak production of aMT6s occurred 9 hours later in night compared with morning shifts, though with a similar mesor indicating adaptation. The adjusted mesor varied somewhat by age group and other personal characteristics, and was somewhat lower among those with higher AM and GM ELF-MF levels (i.e. $\geq 50$ th or 75 th percentile), with differences ranging from $0.94-1.38 \mathrm{ng} / \mathrm{mg}$ creatinine/hour in the morning to $0.96-4.33$ in the night shift observed, with some differences significant.

Conclusions Overall, small differences in mean aMT6s levels were observed according to ELF-MF. Workers during the night shift adapted, showing a delay in the peak time of aMT6s production compared to the day shift. 